\title{
Evidence for an implicit influence of memory on future thinking
}

\author{
KaRL K. SzPUnAR \\ Rotman Research Institute of Baycrest Centre, Toronto, Ontario, Canada
}

\begin{abstract}
The capacity to think about specific events that one might encounter in the future-episodic future thoughtinvolves the flexible (re)organization of memory. The present study demonstrates that implicit processes play an important role here. In two experiments $(N=180)$, participants were asked to generate a personal event that they expected to plausibly occur in the following week. The content of the participants' responses was biased (i.e., primed) by recent thoughts about a specific category of experiences. For instance, participants who had recently been induced to think about social experiences, in the context of an ostensibly unrelated task, were more likely than nonprimed participants to generate similar events occurring in their immediate future. Importantly, the participants were unaware of this unintentional influence of memory. The theoretical and practical implications of these findings for understanding episodic future thought and its relation to memory are discussed.
\end{abstract}

The capacity to think about specific events that one might encounter in the future - episodic future thought - is currently attracting a great deal of attention in both psychology and neuroscience (Atance \& O’Neill, 2001; Schacter \& Addis, 2007; Schacter, Addis, \& Buckner, 2007, 2008; Suddendorf \& Corballis, 2007; Szpunar, 2010). Much of this attention has been focused on Schacter and Addis's (2007) claim that thoughts about one's future involve constructive episodic simulation, the sampling of one's past experiences (i.e., episodic memory) in the service of generating novel scenarios. Indeed, a considerable amount of evidence from neuroimaging, neuropsychology, clinical psychology, and developmental psychology supports this hypothesis.

For instance, research from the neuroimaging literature has revealed a striking similarity in the neural activity that characterizes episodic future thought and episodic memory (Addis, Wong, \& Schacter, 2007; Botzung, Denkova, \& Manning, 2008; Okuda et al., 2003; Szpunar, Watson, $\&$ McDermott, 2007). This finding has been taken as evidence that similar processes underlie the two abilities (Buckner \& Carroll, 2007; Hassabis \& Maguire, 2007; Schacter \& Addis, 2007; Spreng, Mar, \& Kim, 2009). Of particular interest are posterior cortical regions (e.g., posterior cingulate cortex, parahippocampal cortex, and hippocampus) that have been consistently shown to contribute to the retrieval of memories from one's past (Cabeza \& St. Jacques, 2007; Maguire, 2001; McDermott, Szpunar, \& Christ, 2009; Svoboda, McKinnon, \& Levine, 2006). That episodic future thought engages these regions in a similar manner as remembering suggests that the contents of personal memories may in fact be accessed as participants think about their future (Schacter \& Addis, 2009; Szpunar, Chan, \& McDermott, 2009).
Importantly, patient populations who lack or have impoverished episodic memory exhibit an accompanying deficit in episodic future thought. This pattern of impairment has been identified in brain-damaged amnesic patients (Hassabis, Kumaran, Vann, \& Maguire, 2007; Klein, Loftus, \& Kihlstrom, 2002; Rosenbaum, Gilboa, Levine, Winocur, \& Moscovitch, 2009; Tulving, 1985), suicidally depressed individuals (Williams et al., 1996), patients with schizophrenia (D'Argembeau, Raffard, \& Van der Linden, 2008), children under the age of 5 years (Atance \& O'Neill, 2005; Busby \& Suddendorf, 2005), older adults (Addis, Wong, \& Schacter, 2008), and patients with mild Alzheimer's disease (Addis, Sacchetti, Ally, Budson, \& Schacter, 2009). Taken together, the evidence suggests a close relation between episodic future thought and episodic memory.

Accordingly, the emerging literature on episodic future thought has continued to be focused on delineating the contribution of episodic memory, much to the exclusion of other influences of memory that may be worth considering. The purpose of the present study was to elucidate one such unexamined influence - namely, that of implicit memory. Although personal experiences provide the framework around which thoughts about the future are structured, to what extent is the past's influence on the future available to conscious awareness? That is, are we always aware of the manner in which past experiences influence our thoughts about the future? Need we be? The present study will tackle these novel questions in three ways. First, the task of requiring people to generate a personal future episode is considered in some detail. Second, the possibility that memory may impart an implicit or unintentional influence over the outcome of such a task 
is discussed. Finally, two experiments are reported that were specifically designed to examine whether memory imparts an implicit influence on episodic future thought.

\section{Task Analysis}

In the typical experiment designed to examine episodic future thought, participants are presented with a series of words (e.g., dress), time frames (e.g., next week), events (e.g., birthday), or some combination thereof and are asked to use each individual cue to help them think about a personal scenario that they expect to plausibly occur in the future. Each of these cues is open ended, in the sense that the participants are allowed to think about any event that comes to mind so long as they use the cues as a starting point. For instance, consider the cue next week. Although one may conceivably think about a number of hypothetical scenarios related to this time frame, it is typically instructed that only a single event be reported. As was alluded to in the previous section, it is relatively well established that participants will draw on their prior experiences when generating such a scenario. Furthermore, research that has identified knowledge accessibility as a basic cognitive mechanism (Anderson, 1983, 1990, 1993, 1996; see also Anderson \& Matessa, 1998; Anderson \& Schooler, 2000) suggests that the precise content of a participant's response will likely reflect the scenario that comes to mind most easily (see also Kahneman \& Tversky, 1982). For instance, if a participant had recently been thinking about studying for an upcoming exam prior to being asked what he or she will do next week, that participant would be likely to generate a scenario related to that specific context (i.e., that scenario would have relatively higher prior odds, in Bayesian terms, of being reported than other scenarios that have not benefited from recent thought).

What has not yet been considered is whether recent thoughts or experiences can influence episodic future thought in an unintentional manner. That is, given that a cue to think about one's future may potentially elicit a number of responses and that the actual response of the participant will likely reflect currently accessible information, might this accessibility bias occur outside of the awareness of the participant? The answer to this question has important implications for our understanding of the ultimate relation(s) between memory and future thinking. Next, the unintentional influence of memory across a variety of domains that elicit an open-ended response is considered and related to the present context of episodic future thought.

\section{Implicit Memory and Knowledge Accessibility}

Over the past 25 years, psychologists have expressed interest in the unintentional influence of memory on task performance. Much of this research has been conducted under the guise of implicit memory, which is revealed when previous experiences facilitate performance on a task that does not require intentional recollection of those experiences (Roediger \& McDermott, 1993; Schacter, 1987). There are three phases in the typical implicit memory experiment: First, participants are exposed to a set of materials (e.g., a list of words, pictures, or sentences).
Second, the participants take part in some sort of unrelated distractor activity. Third, the participants complete an ostensibly unrelated task that is actually designed to assess the extent to which information learned during the first phase (e.g., the word cheetah) facilitates performance during the third phase (e.g., complete the word fragment $c h_{--} t_{-}$with the first word that comes to mind; answer the question What is the fastest animal on the planet?). The enhanced performance in the third phase for items related to previously studied information relative to items unrelated to previously studied information is referred to as priming. Information learned during the first phase may facilitate performance during the third phase if stimuli in the two tasks share perceptual features (e.g., completing the word fragment $c h_{--} t_{--}$as cheetah), stimuli in the two tasks are conceptually related (e.g., answer cheetah to the question What is the fastest animal on the planet?), or some combination of perceptually and conceptually driven processes (for a detailed review, see Roediger, Weldon, \& Challis, 1989). Finally, in order to ensure that the participants do not adopt explicit strategies and that information learned during the first phase exerts its influence on the third phase in an unintentional manner, researchers will often query the participants as to whether they were aware of any relations between the two phases. Only those participants who report being unaware of any such relation, especially at the time of the third phase of the experiment, are subsequently included in data analysis (Schacter, Bowers, \& Booker, 1989).

Of particular interest to the topic of this article are conceptual implicit memory tasks. In the typical conceptual implicit task, participants are required to produce an openended response that is meaningfully related to a presented cue (e.g., produce an answer to a general knowledge question, generate a list of exemplars relevant to a category cue, generate the first word that comes to mind in response to a cue word). As is the case with most studies of implicit memory, the question of interest is whether participants who were previously exposed to a critical piece of information are more likely to incorporate that information into their response at the time of the test. Indeed, this appears to be the case (again, even though the participants are not aware that this is happening). For instance, participants are more likely to correctly answer general knowledge questions if they were exposed to the answer in an unrelated learning phase (e.g., Blaxton, 1989; Challis \& Sidhu, 1993; Hamilton \& Rajaram, 2001). Similarly, participants are more likely to incorporate a critical exemplar (typically of low frequency; e.g., cheetah) during a category instance generation task (e.g., list the first eight animals that come to mind; see Geraci \& Rajaram, 2004; McDermott \& Roediger, 1996; Mulligan, 1997; Rappold \& Hashtroudi, 1991; Srinivas \& Roediger, 1990) if they have been previously exposed to that item.

The idea that knowledge rendered relatively accessible (e.g., as a result of recent exposure) should influence the content of a participant's open-ended response in an unintentional manner has also been reported in other domains. For instance, it has convincingly been demonstrated that forming an impression of a previously unknown individ- 
ual (Higgins, Bargh, \& Lombardi, 1985; Higgins, Rholes, \& Jones, 1977; Srull \& Wyer, 1979, 1980) and generating a sentence to describe a pictured event (Bock, 1986) may be similarly influenced by currently accessible information that participants are not necessarily aware is guiding the content of their response. In each case, the unintentional influence of memory on task performance has been assessed in a manner that is similar to the conceptual implicit memory tasks described above (i.e., using ostensibly unrelated exposure and test phases and querying the participants as to whether they are aware of any relation between the two).

Given the generative nature of the typical episodic future thought task, it is hypothesized that implicit memory processes may play a similar biasing role in the context currently under consideration. Specifically, it is hypothesized that the scenario that a participant generates in response to a given cue to think about his or her future should reflect the influence of relatively accessible information and, more importantly, that the participant need not be aware of this influence. Although such a finding would not reveal anything specific about episodic future thought itself (i.e., the same principle applies to other generative tasks that are not necessarily episodic in nature), it would underscore two important points: that memory processes other than those related to episodic memory are important to consider in relation to episodic future thought and that the extent to which one's recent past colors one's vision of the future deserves attention.

\section{The Present Experiments}

Two experiments were conducted to address the question of interest: Is there a relation between implicit memory processes and episodic future thought? In order to gain some leverage on this issue, a priming paradigm was implemented that bears a family resemblance to those discussed earlier in relation to implicit memory, impression formation, and sentence production.

Specifically, in each of the experiments that will be discussed below, participants were instructed to complete a series of three mental manipulation tasks: constructing meaningful sentences from scrambled arrangements of words, solving math problems, and generating a personal future episode. The cover story was that the experimenter was interested in whether participants who are good at mentally manipulating one type of information (i.e., verbal or nonverbal) are also good at mentally manipulating other types of information. In reality, the sentence construction task did or did not (i.e., in a between-subjects manner) prime the participants to think about a specific category of personal experiences relevant to the third and final episode-generation task. The question of interest, in both experiments, was whether the content of participantgenerated episodes would be implicitly biased by the personally relevant information that the participants had been induced to contemplate during the earlier, ostensibly unrelated, exposure task. The mental-math task simply served as a brief delay between the exposure phase and the episode-generation phase and served to enhance the credibility of the cover story.

\section{EXPERIMENT 1}

Participants were asked to generate a personally relevant future event that they expected to occur on or near their school campus within the next week. The constraints of this episode-generation task were that the event must occur in a specific setting (on or near school campus) and within a specific time frame (next week). As such, the instructions delimited the information that could potentially become incorporated into the episode. Nonetheless, there remain a large number of scenarios that the participants could potentially imagine in relation to this cue. The question of interest was whether the participants would be more likely to generate social (e.g., going to a party) or academic (e.g., going to class) scenarios after they had been primed to think about personal experiences relevant to similar situations (i.e., social or academic) in the context of an earlier task.

\section{Method}

Participants. Thirty undergraduates were recruited for each level of the between-subjects variable (see the Design section), yielding a total of 90 participants for this experiment. Two thirds of the participants in each condition were tested at Washington University in St. Louis, and one third were tested at Iowa State University.

Design. There was one between-subjects variable, with three levels (priming condition: social group, academic group, control group), in this experiment. Notably, a between-subjects design was instantiated (in both experiments) based on well-documented evidence from the social psychological literature that significant effects of priming are difficult to obtain when multiple concepts are primed in a single experimental session (DeCoster \& Claypool, 2004; Förster \& Liberman, 2007).

Materials. A separate set of materials was administered in each of the three phases of the experiment (i.e., sentence construction, math, episode generation). During the sentence-construction phase (Phase 1), the participants generated meaningful sentences in response to scrambled arrangements of words (cf. Costin, 1969; Srull \& Wyer, 1979, 1980). A total of 30 scrambled word cues were used in this experiment (see Appendix A for a complete list of stimuli). Six of these scrambled word cues specified social situations, 6 were related to academic situations, and 18 were control cues that did not converge on a specific situation. During the delay period (Phase 2), the participants were asked to solve 6 multiplication problems [e.g., $(6 \times 13) / 2=$ ?]. Finally, in the episode-generation phase (Phase 3), the participants were asked to construct a personally relevant future event that they expected to occur on or near their school campus within the next week (see Appendix B for condensed set of instructions).

Procedure. The participants were initially informed that this experiment was designed to test their ability to mentally manipulate verbal (sentence construction) and nonverbal (math and episode generation) stimuli. Furthermore, it was explained to them that the experimenter was interested in whether people who are good at mentally manipulating one type of stimuli are also good at mentally manipulating other types of stimuli. Accordingly, the participants were asked to complete each one of the three tasks as quickly and as accurately as possible. Finally, the participants were told that the three tasks would be presented in random order. In fact, the order of task presentation was consistent across participants (i.e., sentence construction, math, episode generation).

In the first phase of the experiment, each participant was presented with 18 (of 30) scrambled word cues. The scrambled word cues appeared one at a time in the center of a computer screen, and each contained 5 words that were arranged in a nonsensical order (e.g., fun the was party boring). The participants were required to use 4 of the words in each cue to generate a sentence that was most 
relevant to their personal experiences (e.g., the party was fun) and to type in their responses as quickly as possible. ${ }^{1}$ Responses were typed in a space provided directly below each cue.

The participants in the social group were presented with 6 scrambled word cues that could be used to generate sentences related to social situations and 12 cues that did not converge on a specific situation. The order of presentation of the scrambled word cues was such that the 6 social cues appeared in Positions 3, 6, 9, 12, 15, and 18 . The remaining 12 positions drew a random selection of 12 of the 18 control cues. ${ }^{2}$ The participants in the academic group completed the experimental task in the same fashion, with the following exception. These participants were presented with 6 scrambled word cues that could be used to generate sentences related to academic situations, rather than social situations (but also appearing in Positions 3, 6, 9, 12,15 , and 18). Finally, the participants in the control group were presented with (in random order) all 18 scrambled word cues that did not converge on a specific situation.

In the second phase, all of the participants were presented with six math problems. These math problems appeared one at a time in the center of a computer screen. The participants were required to answer these problems as quickly as possible (and were allotted $10 \mathrm{sec}$ per problem). Responses were typed in a space provided directly below each question. The function of the math problems was to introduce a sufficiently long distraction between the priming manipulation and the later episode-generation task (as is typical in the priming literature).

In the third phase, all of the participants were asked to construct a personally relevant future event that they expected to occur on or near their school campus within the next week. The participants were instructed that they had 2 min to describe the event in as much detail as possible. Responses were typed in a space provided directly below the cue. ${ }^{3}$

Finally, upon completing the experiment, all of the participants were asked two questions that served as a manipulation check. First, the participants were asked to guess the true purpose of the experiment. Second, the participants were asked to indicate when they initially became aware of this purpose. The data of any participant who claimed that the target (i.e., primed) scenarios influenced the nature of their episodic future thought and who was aware of this influence during the course of the experiment were excluded from analysis. The entire experiment took approximately $30 \mathrm{~min}$ to complete.

\section{Results}

Scoring criteria. All 90 participant-generated episodes were classified as depicting a social, academic, or unrelated scenario by two independent raters who were blind to the experimental conditions. The raters were instructed to score a scenario as social if the depicted event focused on the participant's social interactions with others (e.g., attending a party, eating lunch with a group of friends at school), as academic if the event focused on the participant's academic obligations (e.g., attending class, studying for a test), and as unrelated if the event did not constitute a social or academic activity (e.g., walking around campus, waking up in the morning).

The resulting interrater reliability was high $(\kappa=.91$; Cohen, 1960). Any disagreements between the raters were discussed, and the event was assigned to a single category. This was the case for only five events. Exclusion of these events did not change the general pattern of results, and so these events were included in all subsequent analyses. ${ }^{4}$

Manipulation check. All 90 participants were unaware of the true purpose of the experiment at the time at which they generated their episodic future thought. In fact, once prompted to guess the true purpose of the experiment, only 1 participant was able to do so. This participant was in the academic group. Interestingly, this participant did not generate an episode that was congruent with the primes that he or she had been exposed to earlier (i.e., academically relevant sentences). Since this individual claimed to have been unaware of the purpose until the manipulation check, his or her data were included in all subsequent analyses.

Note that some other participants also thought that the sentence-construction task was related to the episodegeneration task, but for reasons that were unrelated to the purpose of this experiment. For instance, the majority of the participants who posited a relation between the two phases of the experiment guessed that whether the sentences were completed in a positive (e.g., the party was fun) or negative (e.g., the party was boring) manner would influence the emotional content of the future episode that they subsequently generated. They expressed no explicit knowledge in their explanations that they had been exposed to a category of personal experiences that were meant to influence their later responses.

Distribution of events. The distribution of future episodes generated by the participants in the social, academic, and control groups is presented in Figure 1. In order to examine whether recent thoughts about a specific category of personal experiences (i.e., those primed by sentences) shaped the content of participant-generated episodes, the distributions of events generated by the participants in the social and academic groups were separately contrasted against the distribution of events generated by the participants in the control group. The participants in the control group had been exposed to a similar set of control cues but were not primed to contemplate any particular category of personal experiences. Hence, the distribution of events generated by the participants in the control group (in both

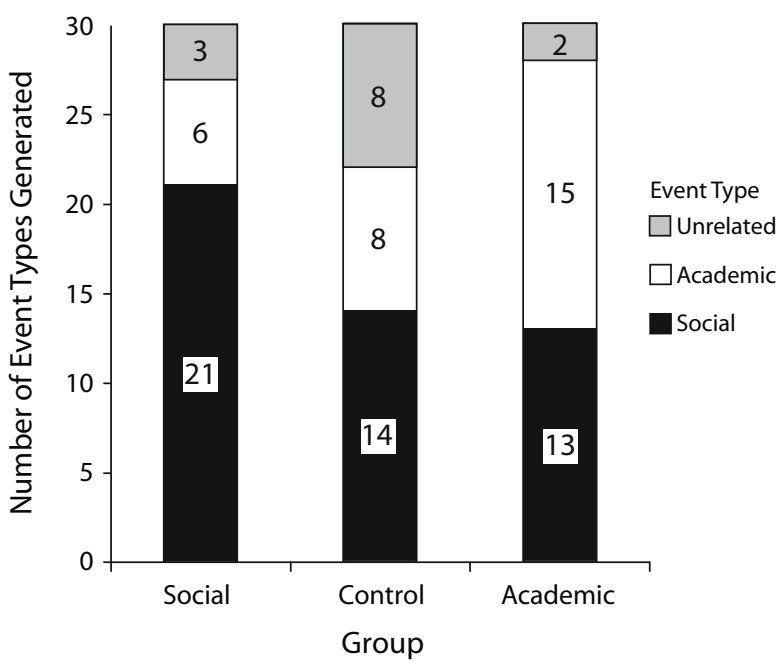

Figure 1. Results of Experiment 1. Total number of event types generated as a function of priming condition. Relative to the control group, the participants in the social group generated more socially relevant future thoughts. Similarly, the participants in the academic group generated more academically relevant future thoughts than did those in the control group. 
experiments) served as an unbiased baseline against which the influence of priming various experiences (social and academic in Experiment 1) could be compared.

First, a chi-square goodness-of-fit test indicated that the distribution of events generated by the participants in the social group (i.e., 21 social scenarios, 6 academic scenarios, and 3 unrelated scenarios) differed significantly from the distribution of events generated by the participants in the control group (i.e., 14 social scenarios, 8 academic scenarios, and 8 unrelated scenarios) $\left[\chi^{2}(2,30)=7.13\right.$, $p=.028]$. This analysis was followed by three a priori contrasts that separately examined the difference in proportions of social, academic, and unrelated events generated by the social and control groups. Two comparisons reached significance. Critically, the participants in the social group were more likely to generate a socially relevant scenario than those in the control group $(Z=1.87, p=$ .031). Furthermore, the participants in the control group were more likely to generate an unrelated scenario than those in the social group $(Z=1.77, p=.038)$.

A second chi-square goodness-of-fit test indicated that the distribution of events generated by the participants in the academic group (i.e., 13 social scenarios, 15 academic scenarios, and 2 unrelated scenarios) also differed significantly from the distribution of events generated by those in the control group (i.e., 14 social scenarios, 8 academic scenarios, and 8 unrelated scenarios) $\left[\chi^{2}(2,30)=10.69\right.$, $p=.005]$. This analysis was also followed by three a priori contrasts that separately examined the difference in proportions of social, academic, and unrelated events generated by the academic and control groups. Two comparisons reached significance. Critically, the participants in the academic group were more likely to generate an academically relevant scenario than those in the control group $(Z=$ $2.47, p=.007)$. Furthermore, the participants in the control group were more likely to generate an unrelated scenario than those in the academic group $(Z=2.12, p=.017)$.

\section{Discussion}

These results clearly demonstrate a relation between implicit memory processes and episodic future thought. The participants in the social and academic groups were more likely than would be expected by chance to generate future episodes that were congruent with the specific category of personal experiences that they had recently been induced to contemplate in the context of a sentence-construction task (i.e., social and academic situations, respectively). More importantly, the participants were unaware of the influence of this particular experimental manipulation.

\section{EXPERIMENT 2}

The purpose of Experiment 2 was to replicate and extend the findings from Experiment 1. In Experiment 1, episodic future thought was constrained to a specific setting (on or near school campus) and time frame (next week). In Experiment 2, an additional constraint was introduced into the instructions, specifying the nature of the episode-generation task. Specifically, the participants in this experiment were asked to construct a personally rel- evant future event that they expected to occur on or near their school campus within the next week and that was related to an academic situation. That is, the episode was constrained to a specific setting (on or near school campus), a specific time frame (next week), and a specific activity (academic). Although this additional constraint should further delimit the information that is applicable to the episode, there still exist a large number of alternative scenarios that one could potentially generate in response to this cue. The question of interest was whether the participants would be more likely to generate an academically related scenario occurring in the context of a classroom (e.g., attending a lecture) or outside the classroom (e.g., studying in the library) after they had been primed to think about personal experiences relevant to similar situations (i.e., academic situations occurring inside or outside the classroom) in the context of an earlier task.

\section{Method}

Participants. Thirty undergraduates were recruited for each level of the between-subjects variable, yielding a total of 90 participants for this experiment. Two thirds of the participants in each condition were tested at Washington University, and one third were tested at Iowa State University.

Design. There was one between-subjects variable, with three levels (priming condition: classroom group, study group, control group), in this experiment.

Materials. A separate set of materials was administered in each of the three phases of the experiment (i.e., sentence construction, math, episode generation). During the sentence-construction phase (Phase 1), the participants generated meaningful sentences in response to scrambled arrangements of words. A total of 30 scrambled word cues were used in this experiment (see Appendix A for a complete list of stimuli). Six of these scrambled word cues specified classroom situations, 6 were related to studying outside the classroom, and 18 were control cues that did not converge on a specific situation (same control cues as in Experiment 1). During the delay period (Phase 2), the participants were asked to solve 6 math problems [e.g., $(6 \times 13) / 2=$ ?] (the same problems as in Experiment 1). Finally, in the episode-generation phase (Phase 3), the participants were asked to construct a personally relevant future event that they expected to occur on or near their school campus within the next week and that was related to an academic situation (see Appendix B for a condensed set of instructions).

Procedure. The procedure of Experiment 2 was identical to that of Experiment 1, with the following exceptions. The participants in the classroom group were presented with 6 scrambled word cues that could be used to generate sentences related to academic situations occurring in the classroom, and the participants in the study group were presented with 6 scrambled word cues that could be used to generate sentences related to academic situations occurring outside the classroom (the participants in both groups were also presented with 12 scrambled word cues that did not converge on a specific situation). As was the case in Experiment 1, the participants in the control group were presented with (in random order) all 18 scrambled word cues that did not converge on a specific situation. After the intervening delay phase (i.e., math problems), the participants in all three groups were asked to construct a personally relevant future event that they expected to occur on or near their school campus within the next week and that was related to an academic situation. The entire experiment, including the manipulation check, took approximately $30 \mathrm{~min}$ to complete.

\section{Results}

Scoring criteria. All 90 participant-generated episodes were classified as depicting an academic activity 
occurring inside the classroom, an academic activity occurring outside the classroom, or an unrelated scenario by two independent raters who were blind to the experimental conditions. The raters were instructed to score a scenario as classroom if the depicted event took place in the context of an academic class (e.g., listening to a lecture, taking a test), as study if the event involved preparation for an upcoming academic obligation (e.g., studying in the library or at home), and as unrelated if the event did not constitute an academic activity occurring inside or outside the classroom (e.g., walking between classes, purchasing materials for class).

The resulting interrater reliability was high $(\kappa=.95)$. Any disagreements between the raters were discussed, and the event was assigned to a single category. This was the case for only three events. Exclusion of these events did not change the general pattern of results, and so these events were included in all subsequent analyses. ${ }^{5}$

Manipulation check. All 90 participants were unaware of the true purpose of the experiment at the time at which they generated their episodic future thought. In fact, once prompted to guess the true purpose of the experiment, only 1 participant was able to do so. This participant was in the classroom group. As was the case in Experiment 1, this participant did not generate an episode that was congruent with the primes that he or she had been exposed to earlier (i.e., sentences related to academic situations occurring in the classroom). Since this individual claimed to have been unaware of the purpose until the manipulation check, his or her data were included in all subsequent analyses. As with Experiment 1, some other participants posited a potential relation between the sentence-construction and episode-generation tasks. None of them, however, demonstrated any inclination that this relation involved a specific category of personal experiences that was primed by the experimenter.

Distribution of events. The distribution of future episodes generated by the participants in the classroom, study, and control groups is presented in Figure 2. In order to examine whether recent thoughts about a specific category of personal experiences (i.e., those primed by sentences) shaped the content of participant-generated episodes, the distributions of events generated by the participants in the classroom and study groups were separately contrasted against the distribution of events generated by the participants in the control group.

First, a chi-square goodness-of-fit test indicated that the distribution of events generated by the participants in the classroom group (i.e., 10 classroom scenarios, 9 study scenarios, and 11 unrelated scenarios) differed significantly from the distribution of events generated by the participants in the control group (i.e., 5 classroom scenarios, 11 study scenarios, and 14 unrelated scenarios) $\left[\chi^{2}(2,30)=6.01\right.$, $p=.049]$. This analysis was followed with three a priori contrasts that separately examined the difference in proportions of classroom, study, and unrelated events generated by the classroom and control groups. Critically, one comparison reached significance, such that the participants in the classroom group were more likely to generate an aca-

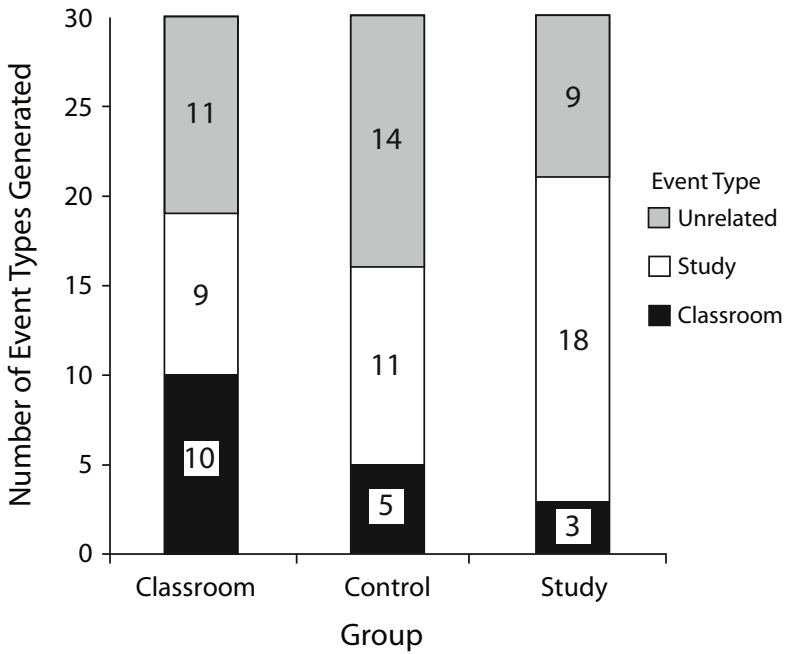

Figure 2. Results of Experiment 2. Total number of event types generated as a function of priming condition. Relative to the control group, the participants in the classroom group generated more academically relevant future thoughts occurring in the classroom. Similarly, the participants in the study group generated more academically relevant future thoughts occurring outside the classroom than did those in the control group.

demically relevant scenario occurring in the classroom than those in the control group $(Z=2.23, p=.013)$.

A second chi-square goodness-of-fit test indicated that the distribution of events generated by the participants in the study group (i.e., 3 classroom scenarios, 18 study scenarios, and 9 unrelated scenarios) also differed significantly from the distribution of events generated by the control group (i.e., 5 classroom scenarios, 11 study scenarios, and 14 unrelated scenarios) $\left[\chi^{2}(2,30)=7.04, p=\right.$ $.029]$. This analysis was also followed by three a priori contrasts that separately examined the difference in proportions of classroom, study, and unrelated events generated by the study and control groups. Critically, one comparison reached significance, such that the participants in the study group were more likely to generate an academically relevant scenario occurring outside the classroom than those in the control group $(Z=2.11, p=.017)$.

\section{Discussion}

The results of this experiment are important in two respects. First, the results of Experiment 2 serve as a conceptual replication of the findings obtained in Experiment 1. Second, these results provide further evidence that there exists a relation between implicit memory processes and episodic future thought. The participants in the classroom and study groups were more likely than would be expected by chance to generate future episodes that were congruent with the specific category of personal experiences that they had recently been induced to contemplate in the context of a sentence-construction task (i.e., academic situations occurring within and outside the classroom, respectively). More importantly, the participants were once again unaware of the influence of this particular experimental manipulation. 


\section{GENERAL DISCUSSION}

The present experiments clearly demonstrate a relation between implicit memory processes and episodic future thought and are notable in two respects. First, the contents of one's thoughts about one's personal future appear to be, at least in part, influenced by recent thoughts that are in a relatively accessible state. Second, and more importantly, one need not be aware of this influence. Next, these findings are related to the relevant literature, and their broader implications are discussed.

\section{What Is Being Primed?}

In both experiments, the participants were asked to use scrambled arrangements of words (e.g., fun the was party boring) to construct sentences that were most relevant to their personal experiences (e.g., the party was fun vs. the party was boring). How is it that this task was so effective in shaping the content of a personal future episode? Some insight was gained from an informal postexperiment interview. ${ }^{6}$ Specifically, the participants tended to report that they followed instructions closely and used whatever personal experiences came to mind to help them decide how to complete each sentence (e.g., deciding whether a fun or boring party was more pertinent to them). Prior research has shown that similar events take place in one's recent past and future (D'Argembeau \& Van der Linden, 2004; Spreng \& Levine, 2006; Szpunar \& McDermott, 2008). Hence, recently thought-about, and thus accessible, experiences may provide a particularly useful source of information for constructing episodic future thoughts.

Of course, that the participants did bring personal experiences to mind does not rule out the possibility that simply constructing meaningful sentences, in the absence of any instructions to bring to mind personal experiences, would have resulted in a similar pattern of data. As is the case with any new area of research (in this case, future thinking), the answer to one question (in this case, the role of implicit memory) opens the door for many others, and future studies will be required to further elucidate the present set of results. For instance, one might also wonder whether priming episodic future thought with pictures rather than sentences would produce similar or perhaps even stronger effects (given that imagery is often associated with this task). Finally, note that with further probing, the participants might have potentially become aware of the experimental manipulation. However, that the participants were not aware of the manipulation during the course of the experiment suggests that an unintentional influence of memory on episodic future thought was indeed demonstrated.

\section{Theoretical Implications: \\ Memory and Future Thought}

According to the prevailing framework of constructive episodic simulation (Schacter \& Addis, 2007), people are thought to sample the contents of personal memories in order to construct novel scenarios that may plausibly occur in the future. The data presented here suggest that the influence of implicit memory will also require serious consider- ation in relation to the emerging concept of episodic future thought. The role of implicit memory, however, can be understood as decidedly different than that of episodic memory. As was alluded to above, episodic memory provides (potentially) much of the content that people are able to draw from when constructing their future (but see Hegdé, 2007, and Szpunar, 2010, who argued for a similar role for more abstracted, nonepisodic, representations). The role of implicit memory, on the other hand, has more to do with the manner in which this personal knowledge (whether it is episodic or not) is utilized. In particular, the present set of experiments demonstrate that one may not necessarily be aware of the influence of relatively accessible information on the content of one's personal thoughts about the future.

On the basis of prior research on conceptual implicit priming and related domains, it is clear that the priming effects obtained here are not specific to episodic future thought but are, rather, related to the generative nature of the episodic future thought task employed. Nonetheless, that thoughts regarding one's future may be implicitly biased has far-reaching implications. Specifically, unlike various conceptual implicit tasks that may arguably lack ecological validity (e.g., name the first eight animals that come to mind), episodic future thoughts are often used as valuable sources of information to guide action (Suddendorf \& Corballis, 1997, 2007; Taylor, Pham, Rivkin, \& Armor, 1998; Taylor \& Schneider, 1989).

\section{Practical Implications}

On the basis of the present findings, one might predict that recent experiences (e.g., positive vs. negative ones) might make it more likely for someone to imagine a related future experience in a similar light and then to approach that upcoming event accordingly - all the while being unaware of the influence of the recent experience. Of course, the extreme example of such a state of affairs occurs in depression. People afflicted with this disorder become overwhelmed by negative thoughts and find it difficult to imagine - and, hence, approach - their future in a positive light (see Williams et al., 1996). The present results suggest that a similar but more subtle phenomenon influences the construction of personal future episodes in healthy adults.

One important implication is that although recent experiences may implicitly bias the content of one's thoughts about the future, changing those experiences may accordingly change the nature of the unintentional influence. Indeed, Williams et al. (1996) showed that healthy adults can be induced to think about their future in a vivid or impoverished manner depending on whether they had recently remembered specific or generic memories, respectively. The participants in this experiment were also unaware of the influence of prior retrieval on future imagination. Of course, one's daily experiences are not always under one's control. Hence, it will also be important for followup studies to examine whether making people aware of the potential influence of recent experiences on future thought might potentially help them to avoid any negative consequences (e.g., realizing that one's negative view of an upcoming event is based largely on the current mood that has been induced by a recent negative experience). 
In addition, it will be important to examine the time course of this implicit influence on episodic future thought. One promising approach might be to examine the relation between how frequently some information is primed (assuming that the participant remains unaware of its influence) and the length of delay between the priming (i.e., exposure) episode and later episode-generation task (cf. Higgins et al., 1985; Lombardi, Higgins, \& Bargh, 1987). However, regardless of the outcome of such studies, the present results suggest that this priming effect in episodic future thought lasts at least $1 \mathrm{~min}$ (the time it took the participants to complete the delay task). In daily life, that represents a reasonable amount of time that may pass between the occurrence of a thought or event that may influence the content of one's thought regarding a novel scenario.

\section{Concluding Remarks}

The present findings are meant to represent a step toward a more complete understanding of the capacity for the human mind/brain to flexibly (re)organize memory in the construction of personal future episodes. In this regard, the present set of experiments clearly demonstrate that implicit memory processes contribute a significant role to the construction of episodic future thought.

\section{AUTHOR NOTE}

The experiments reported in this article were conducted as part of the author's dissertation work at Washington University in St. Louis. The manuscript benefited greatly from comments provided by Kathleen McDermott, Roddy Roediger, Dave Balota, Carl Craver, Pascal Boyer, and Endel Tulving. The author is indebted to Jason Chan for helping to make the data collection possible and to Matt Erdman, Sharda Umanath, and Kaytlin Kopen for help with data collection and scoring. The author is now affiliated with the Department of Psychology at Harvard University (e-mail: karl.szpunar@gmail.com).

\section{REFERENCES}

Addis, D. R., Sacchetti, D. C., Ally, B. A., Budson, A. E., \& Schacter, D. L. (2009). Episodic simulation of future events is impaired in mild Alzheimer's disease. Neuropsychologia, 47, 2660-2671.

Addis, D. R., Wong, A. T., \& Schacter, D. L. (2007). Remembering the past and imagining the future: Common and distinct neural substrates during event construction and elaboration. Neuropsychologia, 45, 1363-1377.

Addis, D. R., Wong, A. T., \& Schacter, D. L. (2008). Age-related changes in the episodic simulation of future events. Psychological Science, 19, 33-41.

Anderson, J. R. (1983). The architecture of cognition. Cambridge, MA: Harvard University Press.

ANDERSON, J. R. (1990). The adaptive character of thought. Hillsdale, NJ: Erlbaum.

ANDERSON, J. R. (1993). Rules of the mind. Hillsdale, NJ: Erlbaum.

Anderson, J. R. (1996). ACT: A simple theory of complex cognition. American Psychologist, 51, 355-365.

Anderson, J. R., \& Matessa, M. (1998). The rational analysis of categorization and the ACT-R architecture. In M. Oaksford \& N. Chater (Eds.), Rational models of cognition (pp. 197-217). Oxford: Oxford University Press.

Anderson, J. R., \& Schooler, L. J. (2000). The adaptive nature of memory. In E. Tulving \& F. I. M. Craik (Eds.), Oxford handbook of memory (pp. 557-570). New York: Oxford University Press.

Atance, C. M., \& O’Neill, D. K. (2001). Episodic future thinking. Trends in Cognitive Sciences, 5, 533-539.

Atance, C. M., \& O'Neill, D. K. (2005). The emergence of episodic future thinking in humans. Learning \& Motivation, 36, 126-144.
Blaxton, T. A. (1989). Investigating dissociations among memory measures: Support for a transfer-appropriate processing framework. Journal of Experimental Psychology: Learning, Memory, \& Cognition, 15, 657-668.

Bock, J. K. (1986). Meaning, sound, and syntax: Lexical priming in sentence production. Journal of Experimental Psychology: Learning, Memory, \& Cognition, 12, 575-586.

Botzung, A., Denkova, E., \& Manning, L. (2008). Experiencing past and future events: Functional neuroimaging evidence on the neural bases of mental time travel. Brain \& Cognition, 66, 202-212.

Buckner, R. L., \& Carroll, D. C. (2007). Self-projection and the brain. Trends in Cognitive Sciences, 11, 49-57.

Busby, J., \& Suddendorf, T. (2005). Recalling yesterday and predicting tomorrow. Cognitive Development, 20, 362-372.

CABeza, R., \& ST. JACQUeS, P. (2007). Functional neuroimaging of autobiographical memory. Trends in Cognitive Sciences, 11, 219-227.

Challis, B. H., \& SidHu, R. (1993). Dissociative effect of massed repetition on implicit and explicit measures of memory. Journal of Experimental Psychology: Learning, Memory, \& Cognition, 19, 115-127.

CoHEN, J. (1960). A coefficient of agreement for nominal scales. Educational \& Psychological Measurement, 20, 37-46.

Costin, F. (1969). The scrambled sentence test: A group measure of hostility. Educational \& Psychological Measurement, 29, 461-468.

D'Argembeau, A., Raffard, S., \& VAn der Linden, M. (2008). Remembering the past and imagining the future in schizophrenia. Journal of Abnormal Psychology, 117, 247-251.

D'Argembeau, A., \& Van Der Linden, M. (2004). Phenomenal characteristics associated with projecting oneself back into the past and forward into the future: Influence of valence and temporal distance. Consciousness \& Cognition, 13, 844-858.

DeCoster, J., \& Claypool, H. M. (2004). A meta-analysis of priming effects on impression formation supporting a general model of informational biases. Personality \& Social Psychology Review, 8, 2-27.

FörSTER, J., \& LibERMAN, N. (2007). Knowledge activation. In A. W. Kruglanski \& E. T. Higgins (Eds.), Social psychology: Handbook of basic principles (2nd ed., pp. 201-231). New York: Guilford.

Geraci, L., \& Rajaram, S. (2004). The distinctiveness effect in the absence of conscious recollection: Evidence from conceptual priming. Journal of Memory \& Language, 51, 217-230.

Hamilton, M., \& RaJaram, S. (2001). The concreteness effect in implicit and explicit memory tests. Journal of Memory \& Language, 44, 96-117.

Hassabis, D., Kumaran, D., Vann, S. D., \& Maguire, E. A. (2007). Patients with hippocampal amnesia cannot imagine new experiences. Proceedings of the National Academy of Sciences, 104, 1726-1731.

Hassabis, D., \& Maguire, E. A. (2007). Deconstructing episodic memory with construction. Trends in Cognitive Sciences, 11, 299-306.

Hegdé, J. (2007). Mental time travel sickness and a Bayesian remedy. Behavioral \& Brain Sciences, 30, 323-324.

Higgins, E. T., BARGH, J. A., \& LOMBARDI, W. [J.] (1985). The nature of priming effects on categorization. Journal of Experimental Psychology: Learning, Memory, \& Cognition, 11, 59-69.

Higgins, E. T., Rholes, W. S., \& Jones, C. R. (1977). Category accessibility and impression formation. Journal of Experimental Social Psychology, 13, 141-154.

Kahneman, D., \& TVersky, A. (1982). The simulation heuristic. In D. Kahneman, P. Slovic, \& A. Tversky (Eds.), Judgment under uncertainty: Heuristics and biases (pp. 201-208). Cambridge: Cambridge University Press.

KLein, S. B., Loftus, J., \& Kinlstrom, J. F. (2002). Memory and temporal experience: The effects of episodic memory loss on an amnesic patient's ability to remember the past and imagine the future. Social Cognition, 20, 353-379.

Lombardi, W. J., Higgins, E. T., \& Bargh, J. A. (1987). The role of consciousness in priming effects on categorization: Assimilation versus contrast as a function of awareness of the priming task. Personality \& Social Psychology Bulletin, 13, 411-429.

Maguire, E. A. (2001). Neuroimaging studies of autobiographical event memory. Philosophical Transactions of the Royal Society B, 356, 1441-1451

McDermott, K. B., \& Roediger, H. L., III (1996). Exact and conceptual repetition dissociate conceptual memory tests: Problems for transfer appropriate processing theory. Canadian Journal of Experimental Psychology, 50, 57-71. 
McDermott, K. B., Szpunar, K. K., \& Christ, S. E. (2009). Laboratory-based and autobiographical retrieval tasks differ substantially in their neural substrates. Neuropsychologia, 47, 2290-2298.

Mulligan, N. W. (1997). Attention and implicit memory tests: The effects of varying attentional load on conceptual priming. Memory \& Cognition, 25, 11-17.

Okuda, J., FujiI, T., Ohtake, H., Tsukiura, T., Tanji, K., Suzuki, K., ET AL. (2003). Thinking of the future and past: The roles of the frontal pole and the medial temporal lobes. NeuroImage, 19, 1369-1380.

RAPPold, V. A., \& Hashtroudi, S. (1991). Does organization improve priming? Journal of Experimental Psychology: Learning, Memory, \& Cognition, 17, 103-114.

Roediger, H. L., III, \& McDermott, K. B. (1993). Implicit memory in normal human subjects. In F. Boller \& J. Grafman (Eds.), Handbook of neuropsychology (Vol. 8, pp. 63-131). Amsterdam: Elsevier.

Roediger, H. L., III, Weldon, M. S., \& Challis, B. H. (1989). Explaining dissociations between implicit and explicit measures of retention: A processing account. In H. L. Roediger III \& F. I. M. Craik (Eds.), Varieties of memory and consciousness: Essays in honour of Endel Tulving (pp. 3-41). Hillsdale, NJ: Erlbaum.

Rosenbaum, R. S., Gilboa, A., Levine, B., Winocur, G., \& MoscoVITCH, M. (2009). Amnesia as an impairment of detail generation and binding: Evidence from personal, fictional, and semantic narratives in K.C. Neuropsychologia, 47, 2181-2187.

SCHACTER, D. L. (1987). Implicit memory: History and current status. Journal of Experimental Psychology: Learning, Memory, \& Cognition, 13, 501-518.

SCHACTER, D. L., \& ADDIS, D. R. (2007). The cognitive neuroscience of constructive memory: Remembering the past and imagining the future. Philosophical Transactions of the Royal Society B, 362, 773-786.

SCHACTER, D. L., \& ADDIS, D. R. (2009). On the nature of medial temporal lobe contributions to the constructive simulation of future events. Philosophical Transactions of the Royal Society B, 364, 1245-1253.

Schacter, D. L., AdDis, D. R., \& BuCKNer, R. L. (2007). Remembering the past to imagine the future: The prospective brain. Nature Reviews Neuroscience, 8, 657-661.

Schacter, D. L., AdDis, D. R., \& Buckner, R. L. (2008). Episodic simulation of future events: Concepts, data, and applications. In A. Kingstone \& M. B. Miller (Eds.), The year in cognitive neuroscience 2008 (Annals of the New York Academy of Sciences, Vol. 1124, pp. 39-60). New York: New York Academy of Sciences.

SCHACter, D. L., Bowers, J., \& Booker, J. (1989). Intention, awareness, and implicit memory: The retrieval intentionality criterion. In S. Lewandowsky, J. C. Dunn, \& K. Kirsner (Eds.), Implicit memory: Theoretical issues (pp. 47-65). Hillsdale, NJ: Erlbaum.

Spreng, R. N., \& Levine, B. (2006). The temporal distribution of past and future autobiographical events across the lifespan. Memory \& Cognition, 34, 1644-1651.

Spreng, R. N., Mar, R. A., \& Kim, A. S. N. (2009). The common neural basis of autobiographical memory, prospection, navigation, theory of mind, and the default mode: A quantitative meta-analysis. Journal of Cognitive Neuroscience, 21, 489-510.

Srinivas, K., \& Roediger, H. L., III (1990). Classifying implicit memory tests: Category association and anagram solution. Journal of Memory \& Language, 29, 389-412.

SRULL, T. K., \& WYER, R. S., JR. (1979). The role of category accessibility in the interpretation of information about persons: Some determinants and implications. Journal of Personality \& Social Psychology, 37, 1660-1672.
SRull, T. K., \& Wyer, R. S., JR. (1980). Category accessibility and social perception: Some implications for the study of person memory and interpersonal judgments. Journal of Personality \& Social Psychology, 38, 841-856.

Suddendorf, T., \& Corballis, M. C. (1997). Mental time travel and the evolution of the human mind. Genetic, Social, \& General Psychology Monographs, 123, 133-167.

Suddendorf, T., \& Corballis, M. C. (2007). The evolution of foresight: What is mental time travel, and is it unique to humans? Behavioral \& Brain Sciences, 30, 299-313.

Svoboda, E., McKinnon, M. C., \& Levine, B. (2006). The functional neuroanatomy of autobiographical memory: A meta-analysis. Neuropsychologia, 44, 2189-2208.

SzPUNAR, K. K. (2010). Episodic future thought: An emerging concept. Perspectives on Psychological Science, 5, 142-162.

Szpunar, K. K., Chan, J. C. K., \& McDermott, K. B. (2009). Contextual processing in episodic future thought. Cerebral Cortex, 19, 1539-1548.

SzPunar, K. K., \& McDermott, K. B. (2008). Episodic future thought and its relation to remembering: Evidence from ratings of subjective experience. Consciousness \& Cognition, 17, 330-334.

Szpunar, K. K., Watson, J. M., \& McDermott, K. B. (2007). Neural substrates of envisioning the future. Proceedings of the National Academy of Sciences, 104, 642-647.

Taylor, S. E., Pham, L. B., Rivkin, I. D., \& Armor, D. A. (1998). Harnessing the imagination: Mental simulation, self-regulation, and coping. American Psychologist, 53, 429-439.

TAYLOR, S. E., \& SchNeIDER, S. K. (1989). Coping and the simulation of events. Social Cognition, 7, 174-194.

Tulving, E. (1985). Memory and consciousness. Canadian Psychology, 26, 1-12.

Williams, J. M. G., Ellis, N. C., Tyers, C., Healy, H., Rose, G., \& MACLEOD, A. K. (1996). The specificity of autobiographical memory and imageability of the future. Memory \& Cognition, 24, 116-125.

\section{NOTES}

1. Across the two experiments, the participants spent $6.5 \mathrm{sec}$ per sentence cue (or approximately $2 \mathrm{~min}$ for the task).

2 . These control cues were included as a means of drawing the participants' attention away from the experimental manipulation. A ratio of 2:1 was taken as appropriate on the basis of similarly designed experiments in the social psychological literature (see DeCoster \& Claypool, 2004).

3 . Across the two experiments, the participants averaged approximately four sentences in describing their future scenario (no more than half a page).

4. In order to ensure that all instructions were clear and that the participants were in fact generating personal future episodes, two additional independent raters (blind to the purposes of the study) scored each scenario as either depicting an event that occurs in the future or the past or is atemporal. Both raters scored each scenario as depicting a future event.

5. As was the case with Experiment 1, two additional independent raters (blind to the purposes of this study) agreed that all scenarios generated by the participants were clearly directed at their personal future.

6 . The final 55 participants who were run in each experiment were asked to identify any strategies that they might have engaged in to help them to construct sentences from the scrambled arrangements of words.

\section{APPENDIXA \\ Scrambled Word Cues (and Sample Completions)}

\section{Social Situations-Experiment 1}

(1) movies group the watched television (e.g., the group watched movies)

(2) fun the was party boring (e.g., the party was fun)

(3) played friends cards sports the (e.g., the friends played sports)

(4) lunch the dinner roommates ate (e.g., the roommates ate dinner)

(5) was workout their short long (e.g., their workout was long)

(6) pleasant date was awkward the (e.g., the date was pleasant) 
APPENDIX A (Continued)

\section{Academic Situations-Experiment 1}

(1) teacher the quickly spoke slowly (e.g., the teacher spoke slowly)

(2) interesting lecture the was boring (e.g., the lecture was interesting)

(3) class late the early ended (e.g., the class ended early)

(4) night all studied procrastinated he (e.g., he studied all night)

(5) was homework the easy hard (e.g., the homework was easy)

(6) library the peaceful was noisy (e.g., the library was noisy)

\section{Classroom Situations-Experiment 2}

(1) teacher the quickly spoke slowly (e.g., the teacher spoke quickly)*

(2) interesting lecture the was boring (e.g., the lecture was interesting)*

(3) class late the early ended (e.g., the class ended early)*

(4) took notes she skimpy detailed (e.g., she took detailed notes)

(5) ignored the teacher questions answered (e.g., the teacher answered questions)

(6) student was lecturer the presenting (e.g., the student was presenting)

*Same as in Experiment 1.

\section{Nonclassroom Situations-Experiment 2}

(1) night all studied procrastinated he (e.g., he studied all night)*

(2) was homework the easy hard (e.g., the homework was easy)*

(3) library the peaceful was noisy (e.g., the library was noisy)*

(4) her long short was essay (e.g., her essay was short)

(5) memorized notes she her rehearsed (e.g., she memorized her notes)

(6) interesting the was uninteresting book (e.g., the book was interesting)

*Same as in Experiment 1.

\section{Control Cues-Experiments 1 and 2}

(1) is boy the tall short (e.g., the boy is tall)

(2) sad girl the happy is (e.g., the girl is happy)

(3) rabbit cute is ugly the (e.g., the rabbit is cute)

(4) the rude is kind man (e.g., the man is rude)

(5) is cut the grass green (e.g., the grass is green)

(6) small plane the large is (e.g., the plane is large)

(7) weak chair the is sturdy (e.g., the chair is sturdy)

(8) hard ball is the soft (e.g., the ball is soft)

(9) stopped the suddenly skidded car (e.g., the car stopped suddenly)

(10) fast slow tractor is the (e.g., the tractor is slow)

(11) young woman felt the old (e.g., the woman felt young)

(12) object is the heavy light (e.g., the object is light)

(13) the is old new clock (e.g., the clock is new)

(14) is snake the long short (e.g., the snake is long)

(15) flew kite high far the (e.g., the kite flew high)

(16) orange is yellow boat the (e.g., the boat is yellow)

(17) is window the clean dirty (e.g., the window is clean)

(18) full box is the empty (e.g., the box is full)

\section{APPENDIX B \\ Episode-Generation Instructions}

\section{Experiment 1}

In the space below, please describe an event involving you somewhere on or near campus within the next week. This can be about anything, so long as it involves you somewhere on, or close to, campus. Just describe the first event that comes to mind. Please describe where the event takes place and what is happening.

\section{Experiment 2}

In the space below, please describe an academically relevant event involving you somewhere on or near campus within the next week. This can be about anything, so long as it involves you on, or close to, campus doing something related to your schooling. Just describe the first event that comes to mind. Please describe where the event takes place and what is happening. 